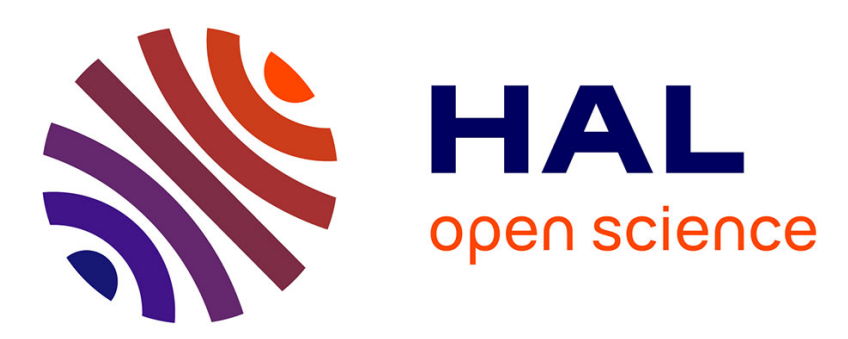

\title{
Fast Simulation of Stent Deployment with Plastic Beam Elements
}

Camille Krewcun, Laurent Sarry, Nicolas Combaret, Emilie Pery

\section{To cite this version:}

Camille Krewcun, Laurent Sarry, Nicolas Combaret, Emilie Pery. Fast Simulation of Stent Deployment with Plastic Beam Elements. EMBC, Jul 2019, Berlin, Germany. hal-02122546

\section{HAL Id: hal-02122546 \\ https://hal.science/hal-02122546}

Submitted on 23 Dec 2019

HAL is a multi-disciplinary open access archive for the deposit and dissemination of scientific research documents, whether they are published or not. The documents may come from teaching and research institutions in France or abroad, or from public or private research centers.
L'archive ouverte pluridisciplinaire HAL, est destinée au dépôt et à la diffusion de documents scientifiques de niveau recherche, publiés ou non, émanant des établissements d'enseignement et de recherche français ou étrangers, des laboratoires publics ou privés. 


\title{
Fast simulation of stent deployment with plastic beam elements
}

\author{
Camille Krewcun ${ }^{1}$, Laurent Sarry ${ }^{1}$, Nicolas Combaret ${ }^{2}$ and Émilie Péry ${ }^{1}$
}

\begin{abstract}
Coronary stent deployment is a reference cardiology intervention, used to treat atherosclerosis and prevent heart attacks. The outcomes of the intervention highly depend on the accuracy of the stent apposition, which could benefit from per-operative prediction tools. In this paper, we propose a fast and mechanically realistic 3D simulation of a coronary stent expansion. Our simulation relies on the finite element method and involves serially linked beam elements to model the slender geometry of a stent. The elements are implemented with a non-linear elasto-plastic behavior, describing realistically the complex deformation of a balloon-expandable stent. As a proof of concept, we simulated the free expansion of a coronary stent. The simulation output was compared with micro-CT data, acquired experimentally during the device expansion. Results show that the plastic beam model is able to reproduce successfully the final geometry of the stent. In addition, the use of 1D elements allows to achieve a significantly lower computational time than for equivalent literature simulations, based on 3D elements. This preliminary work highlights the compatibility of our method with clinical routine in terms of execution time. Further developments include the application of the method to more advanced simulation scenarios, with the addition of a personalized artery model.
\end{abstract}

\section{INTRODUCTION}

The coronary artery disease results from a physiological aging process causing a progressive narrowing (stenosis) of the artery lumen. In the case of coronary arteries, symptoms include an 'acute' phase resulting in myocardial infarction (i.e. heart attack).

In this study, we focus on the Percutaneous Transluminal Coronary Angioplasty (PTCA) procedure with stent deployment. The intervention consists in reducing the stenosis by inflating a balloon and expanding a metallic endoprosthesis (stent). When the balloon is deflated, the stent keeps its deformed shape and stays permanently inside the vessel, to support the artery wall.

The outcomes of this intervention highly depend on the stent geometry and on the accuracy of the deployment. Consequently, numerical simulation is a valuable prediction tool to minimize the probability of complications.

We find in literature an important number of studies using the Finite Element Method (FEM) to simulate the deployment of coronary stents.

A commonly mentioned objective is to study the influence of the stent geometry over the global mechanical behavior,

\footnotetext{
${ }^{1}$ Camille Krewcun, Laurent Sarry, and Émilie Péry are with the Institut Pascal, Université Clermont Auvergne, CNRS, SIGMA Clermont, F-63000 Clermont-Ferrand, France. Email: camille.krewcun@uca.fr

${ }^{2}$ Nicolas Combaret is with the institut Pascal, CHU ClermontFerrand Université Clermont Auvergne, CNRS, SIGMA Clermont, F-63000 Clermont-Ferrand, France.
}

in order to optimize the stent design. Example of such works were published in [1] and [2], in which the free (unconstrained) deployment of a stent is simulated with different geometries. A free expansion is also simulated in [3] in order to study the influence of the balloon model on the global simulation. Studies such as [4] and [5] additionally introduced an artery model in the simulation, allowing to assess the impact of the stent geometry on the artery wall stress. As priority is given to a high precision, almost all the simulated objects in the aforementioned studies (stent, balloon, artery) are discretized finely with volumetric finite elements. Although this allows to optimize the stent design prior to its production, the corresponding models can not be used directly in a clinical environment, for instance to perform patient-specific simulation. Indeed, the computational times required by $3 \mathrm{D}$ element discretization, and standard commercial codes are prohibitive compared to the duration of the corresponding clinical interventions (for instance, an execution time of 48 hours is mentioned in [3]).

In this paper, we propose a faster simulation routine to model accurately the deployment of a balloon-expandable stent. Our objective is to reach an execution time compatible with clinical routine, so that our simulation method could be used in practice as a relevant prediction tool (typically in patient-specific applications).

We decide to base our model on the discretization of the stent geometry by 1D serially linked beam elements. Similar work was proposed by Čanić, Tambača et al. in [6] and [7], where the stent structure is modeled by an assembly of $1 \mathrm{D}$ rod elements. A limitation of these studies is that the proposed mechanical behavior is only elastic, therefore corresponding to auto-expandable stents. Nevertheless, the computational time mention in [6] is significantly faster (with an order of magnitude of $1 \mathrm{~s}$ ).

We decide to use beam elements in a similar way, in order to model the more complex (nonlinear) deformation undergone by a balloon-expandable stent. The use of connected beam elements has already been proved efficient in medical simulation to model slender structures, such as catheters for navigation in [8], endovascular coils for brain aneurysm in [9], flexible needles in [10] and [11], or vascular networks [12]. The use of beam elements in these studies allows to achieve low computational times (up to interactive simulations), but once again is limited to elastic deformations. 


\section{METHOD}

\section{A. Serially-linked beam model}

The first step of our method consists in discretizing the stent structure, by considering its wire-like geometry as a series of straight slender segments. In order to take advantage of this particular shape, we model the stent struts using serially connected beam elements. Beams are discretized into 2 nodes, each described by 6 Degrees Of Freedom (DOFs): three degrees for the node's position, and three for its orientation.

Linking beam elements allows to refine the discretization of the curvilinear stent parts. Typically, the number of beam elements discretizing a given strut length increases with curvature. A limitation of this model is that it is primarily intended to represent simple structures, in which different beam sequences are not connected. In the case of vascular stents, we need to connect more than 3 beam elements at the same node, while conserving the information of each beam's orientation. This particular issue is addressed using Lagrangian constraints, as developed in section II-F.

\section{B. Mesh conception}

Although the various commercialized coronary stents represent a wide range of shapes, all can be decomposed into basic elements. In this way, coronary stents are characterized by two elementary features:

- stent rings are constituted by the periodic repetition of a single geometrical pattern. The dimensions of this particular pattern are sufficient to reconstruct the ring.

- rings are unified in the global stent structure by connectors, which are repeated periodically along the stent length between each pair of rings.

Consequently, only the dimensions of the ring base pattern and the connectors are required to reconstruct the entire stent structure. In order to retrieve these geometric features, we perform a series of X-ray acquisitions on a crimped device. Considering the dimensions of coronary stents (and vascular stents in general), we use micro-CT imaging to obtain a sufficiently high resolution.

With the stent key dimensions, we can reproduce the rings' base pattern and connectors. From these elements, we can take advantage of the aforementioned stent periodicity to simply replicate circumferentially the base pattern, and axially the resulting ring and the connectors. Concretely, the conception is carried out using a Computer Aided Design (CAD) software (Solidworks ${ }^{\mathrm{TM}}$ ). All structures are designed using edges, which can then be converted to straight beam elements. As the association of a beam element to an edge requires to compute the orientation of the edge's nodes, we only use CAD to design a flat version of the global stent mesh. This flat version is then wrapped into a cylindrical shape when the beam elements are created.

\section{Finite Element Method implementation}

Our implementation of the FEM routine relies entirely on the open-source simulation framework SOFA ${ }^{1}$, which was first mentionned in a publication in [13]. The fundamental principle of the simulation workflow in SOFA is to rely on Newton's second law of motion, which can be simply written as:

$$
\mathbf{M} \mathbf{a}_{t+h}=\mathbf{f}_{t+h}(\mathbf{x}, \mathbf{v}),
$$

where $\mathbf{M}$ is the mass matrix of the discretized mechanical system (i.e. if the system is described by $n$ DOFs, then $\mathbf{M}$ is a $n \times n$ matrix), $\mathbf{x}$ is the global position vector, $\mathbf{v}$ the velocity, and a the acceleration. Subscript ${ }_{t}$ indicates that the variable is considered at time $t$, and $h$ is the time step. Adopting the Backward Euler implicit formulation introduced in [14] we obtain the following system:

$$
\underbrace{\left(\mathbf{M}-h \mathbf{B}-h^{2} \mathbf{K}\right)}_{\mathbf{A}} \Delta \mathbf{v}_{t+h}=\underbrace{h \mathbf{f}_{t}(\mathbf{x}, \mathbf{v})+h^{2} \mathbf{K} \mathbf{v}_{t}}_{\mathbf{b}},
$$

where $\mathbf{K}=\frac{\partial \mathbf{f}}{\partial \mathbf{x}}$ is the element stiffness matrix and $\mathbf{B}=\frac{\partial \mathbf{f}}{\partial \mathbf{v}}$ the damping matrix. A more detailed version of the global simulation process can be found in [15]. The last linear system $\left(\mathbf{A} \Delta \mathbf{v}_{t+h}=\mathbf{b}\right)$ is solved in $\Delta \mathbf{v}_{t+h}$ at each time step. From $\Delta \mathbf{v}_{t+h}$ and the Backward Euler scheme, we then retrieve $\mathbf{x}_{t+h}$ making the system progress forward in time.

In (2), we use non-specific expressions for $\mathbf{M}$ and $\mathbf{B}$ : namely a uniform mass model (i.e. $\mathbf{M}$ is diagonal and all its elements are equal), and a Rayleigh damping model (i.e. $\mathbf{B}=r_{M} \mathbf{M}+r_{K} \mathbf{K}, r_{M}$ and $r_{K}$ representing the Rayleigh mass and Rayleigh damping respectively).

On the contrary, the expression of the stiffness matrix $\mathbf{K}$ highly depends on the finite element discretization and mechanical behavior. An important part of this study consists in proposing a novel approach to compute $\mathbf{K}$, using a nonlinear elasto-plastic beam model.

\section{Elasto-plastic behavior}

A critical issue in our study is to handle correctly the complex deformation undergone by balloon expandable stents. During balloon inflation, endoprostheses are deformed from a crimped rest configuration to comply to the deploying balloon membrane. When the balloon is deflated, internal stresses resulting from the deformation do not tend to bring the stent back to its rest shape. This phenomenon indicates a highly plastic deformation, which must be appropriately simulated.

In the following, we describe briefly the main principles in the implementation of a plastic beam model, by focusing on the computation of the associated stiffness matrix $\mathbf{K}$. For more details on the application of the FEM to beam theory, or in the definition of $\mathbf{K}$, we refer the reader to [16] or [17].

\footnotetext{
${ }^{1}$ www.sofa-framework.org
} 


\section{Linear elasticity}

In the case of linear elasticity, the stiffness matrix of a single beam element is computed as follows. First, we define as $\Omega(x, y, z)$ the volume represented by the beam element. Coordinates $x, y$, and $z$ represent material coordinates, so that $x \in[0, l], y \in\left[-\frac{h}{2}, \frac{h}{2}\right]$ and $z \in\left[-\frac{w}{2}, \frac{w}{2}\right]$ respectively, where $l$ represents the beam length, and $h$ and $w$ the height and width of its section. We use the approximation that $h$ and $w$ remain constant during the deformation.

We note $\mathbf{u}(x, y, z, t)$ the continuous displacement field in the element. As mentioned in II-A, beams are discretized into 12 DOFs. Thus, we can also define the nodal displacement vector of a beam as $\tilde{\mathbf{u}}=\left(w_{x, i}, w_{y, i}, w_{z, i}, \theta_{x, i}, \theta_{y, i}, \theta_{z, i}\right)$, where $i \in \llbracket 1,2 \rrbracket$ indicates which of the beam nodes the DOFs are referring to.

The relation between $\mathbf{u}$ and $\tilde{\mathbf{u}}$ is described by shape functions, defining the beam continuous medium by interpolation of the nodal DOFs. In this study, we use 3D Timoshenko beam functions, as expressed by Bazoune and Khulief in [18]. The Timoshenko beam model allows notably to take into account shear deformations and to describe more accurately beams for which the section dimensions are comparable to the length [18]. In our case, this will be the case in the stent parts presenting a high curvature. The shape functions can be summarized in the form of a $3 \times 12$ matrix $\mathbf{N}$ giving:

$$
\mathbf{u}(x, y, z, t)=\mathbf{N}(x, y, z) \tilde{\mathbf{u}}(t) .
$$

The expression of $\mathbf{N}$ is explicitly given in [18].

From the displacement field, we can compute the strain tensor $\epsilon$ using the small strain formulation. We represent the second order strain tensor as a $6 \times 1$ vector, using Voigt notation.

$$
\boldsymbol{\epsilon}(x, y, z, t)=\mathbf{S u}(x, y, z, t)
$$

with

$$
\mathbf{S}=\left(\begin{array}{ccc}
\frac{\partial}{\partial x} & 0 & 0 \\
0 & \frac{\partial}{\partial y} & 0 \\
0 & 0 & \frac{\partial}{\partial z} \\
0 & \frac{1}{2} \frac{\partial}{\partial z} & \frac{1}{2} \frac{\partial}{\partial y} \\
\frac{1}{2} \frac{\partial}{\partial z} & 0 & \frac{1}{2} \frac{\partial}{\partial x} \\
\frac{1}{2} \frac{\partial}{\partial y} & \frac{1}{2} \frac{\partial}{\partial x} & 0
\end{array}\right) \quad \text { and } \quad \boldsymbol{\epsilon}=\left(\begin{array}{c}
\epsilon_{x, x} \\
\epsilon_{y, y} \\
\epsilon_{z, z} \\
\epsilon_{y, z} \\
\epsilon_{x, z} \\
\epsilon_{x, y}
\end{array}\right)
$$

Combining (3) and (4), we obtain:

$$
\boldsymbol{\epsilon}(x, y, z, t)=\mathbf{B}_{\mathbf{e}}(x, y, z) \tilde{\mathbf{u}}(t),
$$

where $\mathbf{B}_{\mathbf{e}}=\mathbf{S N}$ is a $6 \times 12$ matrix.

Using Hooke's law for isotropic linear elasticity, we can relate the stress tensor $\sigma$ to the strain:

$$
\boldsymbol{\sigma}(x, y, z, t)=\mathbf{C} \boldsymbol{\epsilon}(x, y, z, t) .
$$

It can be noted that in (7), $\sigma$ and $\epsilon$ are second order tensors, imposing $\mathbf{C}$ to be expressed as a fourth order tensor. Using again Voigt notation, $\epsilon$ and $\sigma$ can be expressed as 6dimensional vectors, while $\mathbf{C}$ is reduced to a $6 \times 6$ matrix. The corresponding expression for $\mathbf{C}$ can be found in [19]. From the Principle of Virtual Work, we can express the beam stiffness matrix $\mathbf{K}$ in integral form, using the quantities above, as:

$$
\mathbf{K}=\int_{\Omega} \mathbf{B}_{\mathbf{e}}^{T} \mathbf{C B}_{\mathbf{e}} \mathrm{d} \Omega .
$$

The resulting $\mathbf{K}$ is a $12 \times 12$ matrix. Detail of the computation from the continuous expression of the Principal of Virtual Work can be found in [20].

We stress out the fact that matrices $\mathbf{B}_{\mathbf{e}}$ and $\mathbf{C}$ are constant over time, meaning that $\mathbf{K}$ could be computed once and for all at the beginning of the simulation. As elements of $\mathbf{B}_{\mathbf{e}}$ are at most second degree polynomials, we could also compute analytically the integral. This is namely used in [8], [9], and [12] to save computational time. Nevertheless this becomes irrelevant when replacing the linear elasticity law by a nonlinear plastic behavior. Consequently, we concretely compute $\mathbf{K}$ by using Gaussian reduced integration over each beam element.

\section{Plasticity}

In plasticity theory, the constitutive law (i.e. the stressstrain relation) can be decomposed into two phases:

- an elastic phase, during which the internal stress depends linearly on the strain, as in (7),

- a plastic phase, during which part of the strain can be distinguished as resulting from plastic energy dissipation, and the stress-strain relation becomes nonlinear.

The transition from the elastic to the plastic phase is described by a yield criterion $f: \mathbb{R}^{6} \rightarrow \mathbb{R}$, defined on the stress space. In this study, we use the Von Mises yield function:

$$
f(\boldsymbol{\sigma})=\sigma_{e}(\boldsymbol{\sigma})-\sigma_{0}
$$

where

$$
\sigma_{e}(\boldsymbol{\sigma})=\left(\sigma_{e 1}+\sigma_{e 2}\right)^{\frac{1}{2}},
$$

with

$$
\sigma_{e 1}=\frac{1}{2}\left[\left(\sigma_{x}-\sigma_{y}\right)^{2}+\left(\sigma_{y}-\sigma_{z}\right)^{2}+\left(\sigma_{z}-\sigma_{x}\right)^{2}\right],
$$

and

$$
\sigma_{e 2}=3\left(\sigma_{x y}^{2}+\sigma_{y z}^{2}+\sigma_{z x}^{2}\right) .
$$

In (9), $\sigma_{0}$ is a material constant referred to as the yield stress. In a 1D tensile test, it corresponds to the stress value above which the material starts to deform plastically. It is determined experimentally for a given material.

The yield criterion is used as follows:

- if $f(\boldsymbol{\sigma})<0$, the stress state $\boldsymbol{\sigma}$ corresponds to an elastic deformation,

- if $f(\boldsymbol{\sigma})>0, \boldsymbol{\sigma}$ is actually an unacceptable stress state, i.e. the deformation can not be considered as elastic anymore. A new realistic stress state must be computed.

The computation of an acceptable plastic stress state relies on the decomposition of the strain into a plastic and an elastic component:

$$
\boldsymbol{\epsilon}=\boldsymbol{\epsilon}^{e l}+\boldsymbol{\epsilon}^{p l} .
$$

In the following, we adopt a model of perfect plasticity, as described in [21]. The term 'perfect' refers to the fact that 
the yield stress $\sigma_{0}$ remains constant during the plastic phase. Consequently, the yield surface described by $f(\boldsymbol{\sigma})=0$ in the stress space also remains unchanged.

The evolution of the plastic strain is described by a flow rule, of the form:

$$
\mathrm{d} \boldsymbol{\epsilon}^{p l}=\mathrm{d} \lambda \frac{\partial g}{\partial \boldsymbol{\sigma}} .
$$

The scalar value $\mathrm{d} \lambda$ is known as the plastic multiplier, and $g: \mathbb{R}^{6} \rightarrow \mathbb{R}$ is a plastic potential defined on the stress space. In our case, we use an associative flow rule, meaning that the plastic potential is considered equal to the yield criterion:

$$
g(\boldsymbol{\sigma})=f(\boldsymbol{\sigma}) .
$$

This approximation is generally acceptable for metals, but gives incoherent results with more complex elasto-plastic materials (such as soil, or concrete).

As the constitutive law becomes nonlinear in plastic phase, it is not possible to use (7) to compute the new stress state at each time step anymore. Additionally, the expression of $\mathbf{K}$ given in (8), relying on the fact that $\mathbf{C}$ is constant, is no longer relevant.

An alternative is to integrate the differential elasto-plastic stress-strain relation, in order to compute the new stress state incrementally, at each time step. A solution for this well known issue is to adopt an integration scheme based on the implicit return algorithm. Although the idea was introduced before, we refer the reader to [21] for a detailed implementation, and to Simo and Taylor's study [22] for a thorough review of this procedure. We give a brief description of the method below.

We want to compute a new stress increment $\Delta \sigma$ so that $\sigma_{t+h}=\sigma_{t}+\Delta \sigma$. The computation is decomposed into two steps. At first, we compute an incremental elastic predictor, as:

$$
\Delta \boldsymbol{\sigma}^{e l}=\mathbf{C} \Delta \boldsymbol{\epsilon}
$$

Depending on the sign of $f\left(\sigma_{t}+\Delta \boldsymbol{\sigma}^{e l}\right)$, we consider two scenarios:

- if $f\left(\boldsymbol{\sigma}_{t}+\Delta \boldsymbol{\sigma}^{e l}\right)<0$, the new stress state $\boldsymbol{\sigma}_{t+h}$ actually results from a purely elastic deformation, and the elastic predictor is accurate. Therefore $\Delta \sigma=\Delta \sigma^{e l}$. This scenario is also chosen if $\nabla^{T} f\left(\boldsymbol{\sigma}_{t}\right) \Delta \boldsymbol{\sigma}^{e l}<0$ (unloading case).

- if $f\left(\boldsymbol{\sigma}_{t}+\Delta \boldsymbol{\sigma}^{e l}\right)>0$, the elastic predictor does not provide an acceptable stress state. We must couple it with a corrective term, in order to obtain a plastic stress state.

As described in [21], the orthogonal return algorithm consists in searching for a corrective stress increment which, summed to the elastic predictor, gives the final stress state $\boldsymbol{\sigma}_{t+h}$. The term 'return' refers to the fact that the direction of this corrective stress increment is given by the unit normal to the yield surface. When using the Von Mises criterion, the regularity of the function implies that this direction is the radial direction. This is known as the radial return algorithm.
As given in [21], we obtain the following update for the new stress state:

$$
\boldsymbol{\sigma}_{t+h}=\frac{\sigma_{0}}{\sigma_{e}\left(\boldsymbol{\sigma}_{t+h}^{e l}\right)} \mathbf{s}_{t+h}^{e l}+\operatorname{Tr}\left(\boldsymbol{\sigma}_{t+h}^{e l}\right) \mathbf{I},
$$

where $\boldsymbol{\sigma}_{t+h}^{e l}=\boldsymbol{\sigma}_{t}+\Delta \boldsymbol{\sigma}^{e l}$ and $\mathbf{s}_{t+h}^{e l}$ is the corresponding deviatoric stress $\left(\mathbf{s}_{t+h}^{e l}=\boldsymbol{\sigma}_{t+h}^{e l}-\operatorname{Tr}\left(\boldsymbol{\sigma}_{t+h}^{e l}\right) \mathbf{I}\right)$. Following the consistency condition $\left(\nabla f^{T} \mathrm{~d} \sigma=0\right)$, the plastic multiplier increment is computed as:

$$
\Delta \lambda=\frac{\nabla f^{T} \boldsymbol{\sigma}_{t+h}^{e l}}{\nabla f^{T} \mathbf{C} \nabla f} .
$$

Once the stress state $\boldsymbol{\sigma}_{t+h}$ is known, we may finally compute the resulting internal forces, and linearized stiffness matrix, so that they are taken into account in the global mechanical system (2).

The new internal forces are simply computed by integration:

$$
\mathbf{f}_{i n t}\left(\boldsymbol{\sigma}_{t+h}\right)=\int_{\Omega} \mathbf{B}_{\mathbf{e}}^{T} \boldsymbol{\sigma}_{t+h} \mathrm{~d} \Omega .
$$

The linearized version of the stiffness matrix (or tangent stiffness matrix) $\mathbf{K}_{t}$ can be expressed in a similar analysis way as for (8), leading to:

$$
\mathbf{K}_{t}=\int_{\Omega} \mathbf{B}_{\mathbf{e}}^{T}\left(\frac{\mathrm{d} \boldsymbol{\sigma}}{\mathrm{d} \boldsymbol{\epsilon}}\right) \mathbf{B}_{\mathbf{e}} \mathrm{d} \Omega .
$$

Starting from Hooke's law, we have:

$$
\begin{aligned}
\mathrm{d} \boldsymbol{\sigma} & =\mathbf{C} \mathrm{d} \boldsymbol{\epsilon}^{e l} \\
& =\mathbf{C}\left(\mathrm{d} \boldsymbol{\epsilon}-\mathrm{d} \boldsymbol{\epsilon}^{p l}\right) \\
\mathrm{d} \boldsymbol{\sigma} & =\mathbf{C}\left(\mathrm{d} \boldsymbol{\epsilon}-\mathrm{d} \lambda \frac{\partial g}{\partial \boldsymbol{\sigma}}\right)
\end{aligned}
$$

Through calculus, and using the consistency condition:

$$
\nabla f^{T} \mathrm{~d} \boldsymbol{\sigma}=0
$$

we obtain an equivalent differential relation between stress and strain in plastic deformation:

$$
\mathrm{d} \boldsymbol{\sigma}=\mathbf{C}^{e p} \mathrm{~d} \boldsymbol{\epsilon}
$$

with

$$
\mathbf{C}^{e p}=\left(\mathbf{C}-\frac{\mathbf{C} \frac{\partial f}{\partial \boldsymbol{\sigma}} \frac{\partial f}{\partial \boldsymbol{\sigma}}^{T} \mathbf{C}}{\frac{\partial f}{\partial \boldsymbol{\sigma}}^{T} \mathbf{C} \frac{\partial f}{\partial \boldsymbol{\sigma}}}\right)
$$

This allows to explicitly express the tangent stiffness as:

$$
\mathbf{K}_{t}=\int_{\Omega} \mathbf{B}_{\mathbf{e}}^{T} \mathbf{C}^{e p} \mathbf{B}_{\mathbf{e}} \mathrm{d} \Omega .
$$




\section{E. Expansion forces}

In order to simulate the balloon expansion, we apply radial forces on each mesh node. These forces are incremented linearly in time, up to a maximal intensity value $f_{i}$ where $i$ represents the index of a node.

We approximate this intensity by considering that the inflation pressure of the balloon is directly transmitted to the stent inner surface $S_{i n t}$. The global force intensity $F$ applied on the stent can then be related to the inflation pressure $P$ by:

$$
F=P \times S_{\text {int }}=P \times w \times h \times l_{\text {tot }},
$$

where $l_{t o t}$ stands for the mesh total length, computed by summing the lengths of all the elements.

Then, we associate to each node a scalar value $\alpha_{i}$ corresponding to the ratio of the lengths of the beam elements it belongs to, over $2 l_{\text {tot }}$ (as each beam length is counted twice: once for each of its nodes). $\alpha_{i}$ gives an estimation of which proportion of the stent length is affected by node $i$. We simply use these coefficients to distribute global force intensity over the nodes, leading to:

$$
f_{i}=\alpha_{i} F .
$$

In order to reproduce the balloon inflation and deflation, we increment and decrement linearly the intensity of the nodal forces, over a given number of time steps. The direction of theses forces remains constant (along radial direction) during the deformation.

\section{F. Constraints}

As mentioned in the beginning of this paper, we need to ensure nodal cohesion between three or more beam elements, in certain parts of the mesh (for instance at stent ring connections). This is achieved by making use of Lagrangian constrained dynamics, as introduced in [23]. This method was specifically described on beam elements in SOFA by Sorokin et al. in [24], to simulate filopodia. Once again, we use the same model without modifications, and refer the reader to the aforementioned article for more details.

Briefly, the addition of constraints involves an additional step in the numerical solution;

- At first, (2) is solved without being constrained, leading to a new position (free motion).

- In a second time, a modified constrained system is solved:

$$
\mathbf{A} \Delta \mathbf{v}_{t+h}=\mathbf{b}+h \mathbf{H}^{T} \boldsymbol{\lambda},
$$

where $\mathbf{H}$ is a Jacobian containing the constraint directions, and $\boldsymbol{\lambda}$ contains the Lagrange multipliers associated to each constraint. In SOFA, this system is typically solved iteratively using a Gauss-Seidel algorithm.

\section{RESULTS}

As a proof of concept, the methodology presented above was entirely carried out on a Presillion Plus (Cordis, Fremont, California, U.S.) coronary stent.

\section{A. Mesh conception}

A first run of micro-CT images was acquired on the crimped stent, to retrieve its geometrical base features. The acquisition was performed with a micro-CT scanner (Explore CT $120^{\circledR}$, GE Healthcare, Chicago, Illinois, U.S.) with a predefined routine of 700 projections. The dimensions of the stent base patterns were measured on the resulting 3D image, and a corresponding flat mesh was conceived accordingly, as shown in Fig. 1.

The studied stent can be decomposed into two sinusoidal ring patterns, used alternatively over a total of 13 rings. The global structure was discretized with 1956 beam elements (2005 nodes), and its cohesion assured with 85 bilateral constraints.

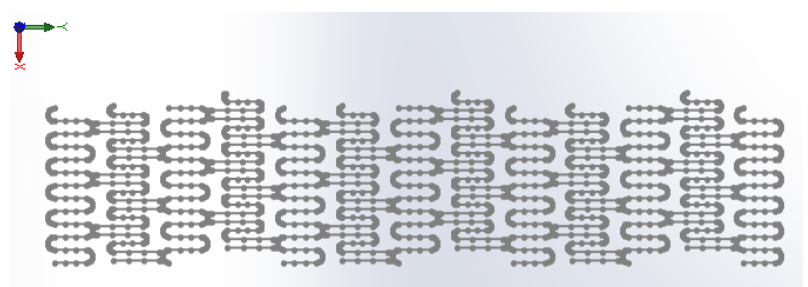

Fig. 1. Flat mesh reproducing the Presillion Plus coronary stent structure. The mesh was conceived with $\mathrm{CAD}$, from micro-CT-acquired geometrical data.

\section{B. Simulation parameters}

The flat mesh was imported into SOFA, and wrapped into a cylindrical shape. The radial expansion forces applied to each node were computed for an inflation pressure of $P=3.0 \mathrm{MPa}$ (about $30 \mathrm{~atm}$ ), and the FEM routine was run with $\Delta t=1 \mu \mathrm{s}$.

The simulation was launched on a common workstation (Intel Xeon E3-1270 v5 (8CPUs) $3.6 \mathrm{GHz}, 16 \mathrm{~GB}$ RAM, Windows10 64 bits), without any form of optimization (single thread CPU execution). The execution time for the whole simulation was around 1 hour.

\section{Experimental validation}

In order to assess the precision of our proof-of-concept model, we performed three other micro-CT acquisitions, on a Presillion Plus deployed with an inflation pressure of 12,16 and 20 atm, respectively. Stent expansion was unconstrained (i.e. no artery phantom was used), and the parameters were the same as for the crimped stent acquisition.

Quantitative evaluation was achieved by comparing specific geometrical features on two pointsets, extracted from the simulation output and from the micro-CT $3 \mathrm{D}$ reconstruction, respectively. The simulation pointset simply corresponds to the mesh nodes, while the micro-CT pointset was extracted by converting the volumetric representation of the stent to a binary skeleton. We performed rigid registration between the two pointsets. In a first step, rigid transform was carried out to superimpose the principal axes of inertia and centers of gravity. In a second step, we used a rigid rotation around the 
common axis to minimize the Euclidean distance between simulated points and their closest neighbors in the CT pointset. 3D rendering of the registration output is given in Fig. 3 for a crimped and a deployed configurations.

The first available feature is the Euclidean distance between each pair of coupled points. We measured an average distance of $0.17 \mathrm{~mm}$, which is approximately 10 times smaller than the macroscopic dimensions of the stent (the diameter and length's order of magnitude being typically $1 \mathrm{~mm}$ ). In the same way, we computed the radial distance between each pair of points. We obtained an average value of $80 \mu \mathrm{m}$, which is the same order of magnitude as the strut thickness (the stent section dimensions being $(50 \times 50) \mu \mathrm{m})$.

The last geometrical feature is the deployment radius, computed for each point as the radial distance with the stent rotation axis. Fig. 2 shows the mean radius in function of the axial position, along the stent centerline. We observe that the expansion radius is accurately estimated by the simulation around 0 , with a minimum difference of $2.29 \times 10^{-3} \mathrm{~mm}$ between the two models, for an axial position of $-0.28 \mathrm{~mm}$. On the contrary, the radius is underestimated at the stent extremities, with a maximum difference of $0.210 \mathrm{~mm}$ for an axial position of $-4.7 \mathrm{~mm}$. We note that the free deployment of the stent leads to an heterogeneous radius along the centerline, which is correctly reproduced by the simulation. In practice, when a coronary stent is deployed inside an artery, its final radius is imposed by the artery wall.

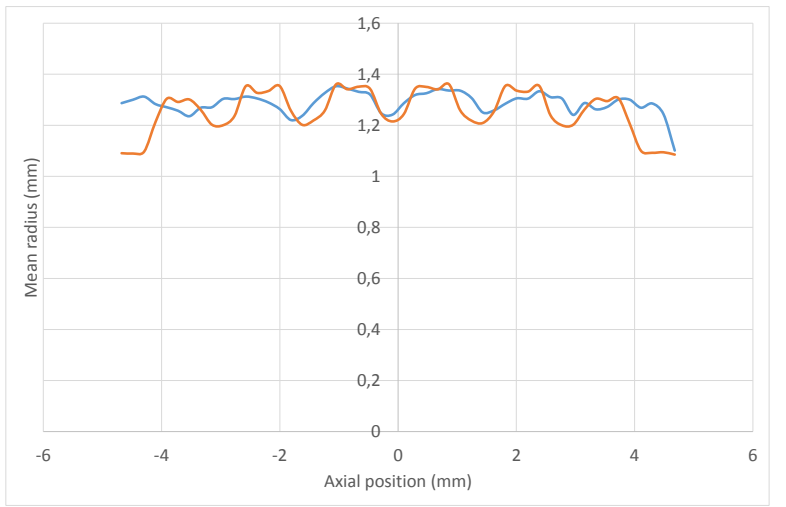

Fig. 2. Comparison of the Presillion Plus expansion radius between the simulation output (orange curve) and a micro-CT ground-truth acquired with $P=12$ atm (blue curve). The result is given in function of the stent axial position, along its rotation axis.

\section{DISCUSSION}

The first point we want to mention is that the model we propose for balloon-expandable stents is able to reproduce the free expansion of a stent, in a reasonable amount of time. The comparison of the simulation output with experimental micro-CT data, based on basic geometrical features, showed that the deployed configuration could be accurately reproduced in terms of radial expansion. In
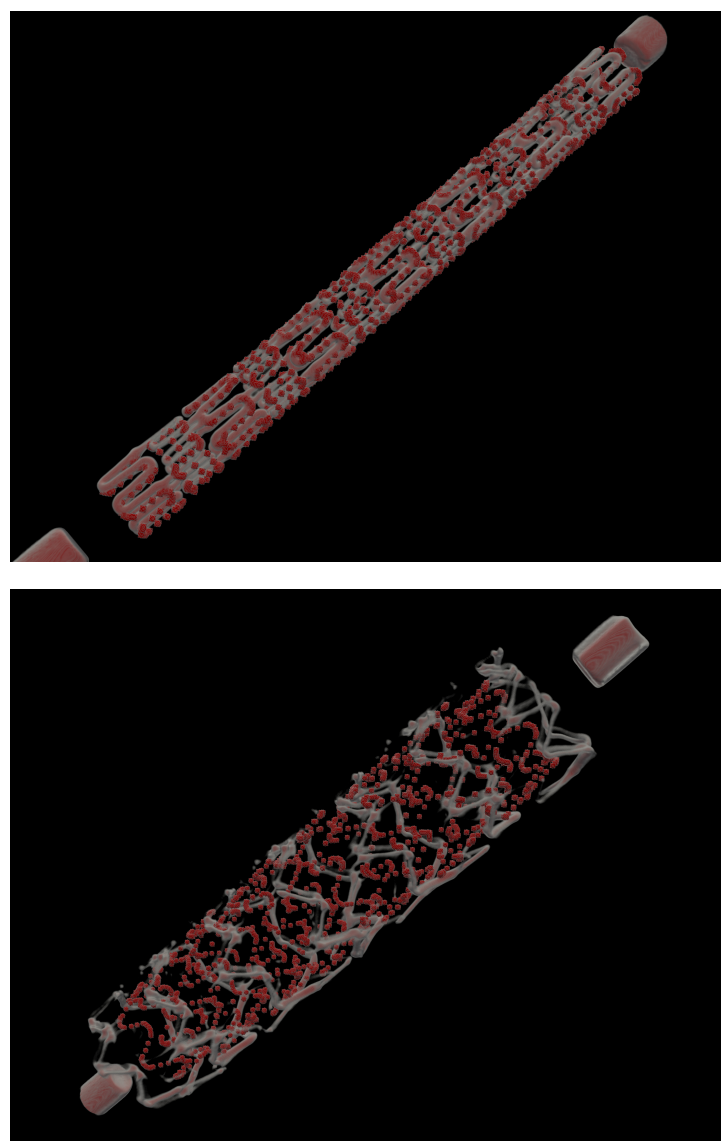

Fig. 3. 3D rendering of the rigid registration between the simulation output and the micro-CT acquisition. The CT volumetric reconstruction is displayed plain and in grey, while the simulation mesh nodes are superimposed as red points. The top image corresponds to the crimped configuration $(P=$ $0 \mathrm{~atm})$, and the bottom image to a deployed configuration $(P=12 \mathrm{~atm}$ in the micro-CT acquisition).

addition, the achieved execution time is significantly smaller than for similar simulations using volumetric elements and standard commercial codes. Among the studies mentioned in introduction and also modeling stent free expansions, but with 3D elements ([1], [2] and [3]), only De Beule et al. in [3] mention an execution time: less than 48 hours, for simulations run with ABAQUS large deformation analysis on a in-house-built high performance computing cluster. Although their discretization is particularly dense, we can expect similar computational times for [1] and [2] in which the simulation also relies on ABAQUS large deformation analysis. Our 1D beam model is likely to be 10 times faster (48 times faster than [3]), while running on a regular workstation (single CPU thread).

One perspective of our work is to run an optimized version of our code on a more powerful computer (Intel Xeon E5-2630 v4 $2.20 \mathrm{GHz}$ (20 cores)). As several tasks can be run in parallel over the beam elements (namely the computation of the internal forces, the tangent stiffness matrix, and plastic stress states), we are confident in being able to divide the execution time by at least 10 . 
Nevertheless, the results also exhibit non-negligible limitations. We can see in the bottom picture of Fig. 3 that the stent length in the final configuration is shorter in the simulation than in the micro-CT imaging $(9.35 \mathrm{~mm}$ against $11.1 \mathrm{~mm})$. Even with similar expansion diameters, this unexpected shortening creates an axial shift in the whole structure. This phenomenon could be explained by various approximations of our model, the most likely of which are listed below.

The perfectly plastic model is an obvious approximation for the plastic behavior. Even if hardening phenomena are not crucial considering the unique loading/unloading cycle undergone by the stent during its deployment, we could straightforwardly extend our model by taking into account the evolution of the yield criterion, as proposed in [21]. Under the same expansion forces, such a modification should notably stabilize the stent deformation in comparison with perfect plasticity.

The balloon simulation is another potential issue. As the balloon inflation and deflation are only simulated through incremental forces, we do not render directly the equilibrium that, in reality, takes place between the balloon elastic membrane and the coronary stent. Typically the implementation of this equilibrium could limit the shortening phenomenon mentioned above.

The last clear limitation regarding our results is the fact that the default implementation of the Newton-Raphson algorithm in SOFA (used to solve (2)) restricts the procedure to 1 iteration per time step. Although this does not represent an issue when dealing with linear elastic behaviors, the introduction of a nonlinear plastic behavior should benefit from a fully iterative procedure.

Although we restricted the accuracy assessment of this proofof-concept study to basic geometrical criteria, future work should ensure that the precision of our model is compatible with clinical use.

\section{CONCLUSION}

In this paper, we present a complete methodology to realistically simulate the plastic deformation of a balloonexpandable stent, in a reasonable time. The efficiency of the algorithm relies on an original discretization of the sent by $1 \mathrm{D}$ serially linked beam elements. Even if this study is focused on coronary stents, we highlight the fact that - to the best of our knowledge - this geometrical representation could be applied to other models of (uncovered) vascular stents, for instance designed to treat the carotid, femoral, or iliac arteries.

Additionally, we experimentally assessed the output of the simulation using basic geometrical criteria, thus establishing the feasibility of the method and the compatibility of our model with clinical routine.

As this work remains preliminary, it is meant to be extended towards more realistic applications. Consequently, the objectives of our future investigations will be the enhancement of the present beam model and the introduction of a constraining artery model in the simulation. Using a personalized model (for instance retrieved from Optical Coherence
Tomography endovascular imaging), the simulation could be integrated in a clinical routine, in the form of a reliable visualization tool.

\section{ACKNOWLEDGMENT}

The authors would like to thank Arnaud Briat and Leslie Mazuel from the Imagerie Moléculaire et Stratégies Théranostiques (IMOST) laboratory (UMR 1240 Inserm / UCA), for their assistance in the acquisition of the micro-CT images.

\section{REFERENCES}

[1] F. Migliavacca, L. Petrini, M. Colombo, F. Auricchio, and R. Pietrabissa, "Mechanical behavior of coronary stents investigated through the finite element method," Journal of Biomechanics, vol. 35, pp. 803811, 2002.

[2] L. Petrini, F. Migliavacca, F. Auricchio, and G. Dubini, "Numerical investigation of the intravascular coronary stent flexibility," Journal of Biomechanics, vol. 37, pp. 495-501, 2004.

[3] M. D. Beule, P. Mortier, S. G. Carlier, B. Verhegghe, R. V. Impe, and P. Verdonck, "Realistic finite element-based stent design: The impact of balloon folding," Journal of Biomechanics, vol. 41, pp. 383-389, 2008.

[4] F. Gijsen, F. Migliavacca, S. Schievano, L. Socci, L. Petrini, A. Thury, J. J. Wentze, A. van der Steen, P. Serruys, and G. Dubini, "Simulation of stent deployment in a realistic human coronary artery," BioMedical Engineering OnLine, vol. 7, p. 23, 2008.

[5] H. Zahedmanesh and C. Lally, "Determination of the influence of stent strut thickness using the finite element method: implications for vascular injury and in-stent restenosis," Medical \& Biological Engineering \& Computing, vol. 47, pp. 385-393, 2009.

[6] J. Tambača, M. Kosor, S. Čanić, and D. P. M.D, "Mathematical modeling of vascular stents," SIAM Journal on Applied Mathematics, vol. 70, pp. 1922-1952, 2010.

[7] S. Čanić and J. Tambača, "Cardiovascular stents as pde nets: 1d vs. 3d," IMA Journal of Applied Mathematics, vol. 77, pp. 748-770, 2012.

[8] C. Duriez, S. Cotin, J. Lenoir, and P. Neumann, "New approaches to catheter navigation for interventional radiology simulation," Computer Aided Surgery, vol. 11, pp. 300-308, 2006.

[9] J. Dequidt, M. Marchal, C. Duriez, E. Kerrien, and S. Cotin, "Interactive simulation of embolization coils: Modeling and experimental validation," in Medical Imaging Computing and Computer Assisted Intervention - MICCAI, 2008.

[10] C. Duriez, C. Guébert, M. Marchal, S. Cotin, and L. Grisoni, "Interactive simulation of flexible needle insertions based on constraint models," in Medical Image Computing and Computer-Assisted Intervention - MICCAI, 2009.

[11] Y. Adagolodjo, L. Goffin, M. D. Mathelin, and H. Courtecuisse, "Inverse real-time finite element simulation for robotic control of flexible needle insertion in deformable tissues," in IEEE/RSJ International Conference on Intelligent Robots and Systems (IROS), 2016.

[12] I. Peterlík, C. Duriez, and S. Cotin, "Modeling and real-time simulation of a vascularized liver tissue," in Medical Imaging Computing and Computer Assisted Intervention - MICCAI, 2012.

[13] J. Allard, S. Cotin, F. Faure, P.-J. Bensoussan, F. Poyer, C. Duriez, H. Delingette, and L. Grisoni, "Sofa - an open source framework for medical simulation," in MMVR 15 - Medicine Meets Virtual Reality, 2007.

[14] D. Baraff and A. Witkin, "Large steps in cloth simulation," in Proceedings of the 25th Annual Conference on Computer Graphics and Interactive Techniques, ser. SIGGRAPH '98, 1998, pp. 43-54.

[15] F. Faure, C. Duriez, H. Delingette, J. Allard, B. Gilles, S. Marchesseau, H. Talbot, H. Courtecuisse, G. Bousquet, I. Peterlík, and S. Cotin, SOFA: A Multi-Model Framework for Interactive Physical Simulation. Berlin, Heidelberg: Springer, 2012, pp. 283-321.

[16] J. S. Przemieniecki, Theory of Matrix Structural Analysis, 1st ed., M.-H. book company, Ed., 1968.

[17] M. Bonnet and A. Frangi, Analyse des solides défomables par la méthode des éléments finis. Éditions de l'École Polytechnique, 2013, ch. 3, 5-7.

[18] A. Bazoune, Y. A. Khulief, and N. G. Stephen, "Shape functions of three dimensional timoshenko beam element," Journal of Sound and Vibration, vol. 259, pp. 473-480, 2003. 
[19] J. S. Przemieniecki, Theory of Matrix Structural Analysis, 1st ed., 1968, pp. 12-17.

[20] — Theory of Matrix Structural Analysis, 1st ed., 1968, ch. 10

[21] T. J. R. Hughes, Theoretical foundation for large-scale computations of nonlinear material behavior, 1984, ch. II.

[22] J. Simo and R. Taylor, "Consistent tangent operators for rateindependent elastoplasticity," Computer Methods in Applied Mechanics and Engineering, vol. 48, pp. 101 - 118, 1985.

[23] A. Witkin. (1997) Physically based modeling: Constrained dynamics. [Online]. Available: https://www.cs.cmu.edu/ baraff/pbm/constraints.pdf

[24] D. Sorokin, I. Peterlík, V. Ulman, D. Svoboda, T. Nečasová, K. Morgaenko, L. Eisellová, L. Tesařová, and M. Maška, "Filogen: A modelbased generator of synthetic $3 \mathrm{~d}$ time-lapse sequences of single motile cells with growing and branching filopodia," IEEE Trans. Med. Imag., vol. 37 , pp. $2630-2641,2018$. 